\title{
Note: The effect of viscosity on the rate of diffusion-limited association of nanoparticles
}

\author{
Vladimir P. Zhdanov \\ Section of Biological Physics, Department of Applied Physics, Chalmers University of Technology, Göteborg, Sweden \\ and Boreskov Institute of Catalysis, Russian Academy of Sciences, Novosibirsk, Russia
}

(Received 23 August 2015; accepted 17 October 2015; published online 29 October 2015)

[http://dx.doi.org/10.1063/1.4934948]

In his seminal article, ${ }^{1}$ Smoluchowski analyzed 3D diffusion-limited association of suspended spherical particles and showed that except a short initial phase the process can be described at the mass-action law level with the rate constant given by

$$
k_{\mathrm{a}}=4 \pi \mathcal{R} \mathcal{D},
$$

where $\mathcal{R}$ is the contact radius or, more specifically, the sum of the radii, and $\mathcal{D}$ is the sum of the diffusion coefficients of the two species. The first important works complementing what was done by Smoluchowski were performed by Collins and Kimball ${ }^{2}$ and Berg. ${ }^{3}$ Numerous subsequent theoretical studies are reviewed in the textbooks on chemical kinetics. The most recent extensions of the conventional treatments are focused on the role of stochasticity ${ }^{4}$ and crowding. ${ }^{5}$

In numerous applications of Eq. (1) or other similar equations to nanoparticles (or, in the context of biophysics, to proteins, micelles, vesicles, virions, ligand-functionalized metal nanoparticles, etc.), the diffusion coefficient, $D$, is usually identified with that predicted by hydrodynamics, $D_{\circ}=k_{\mathrm{B}} T / 6 \pi \eta R$, where $R$ is the particle radius, and $\eta$ is the viscosity coefficient. The latter expression is valid for single particles far from interfaces. During the contacts between particles or with an interface, $D$ is, however, smaller than

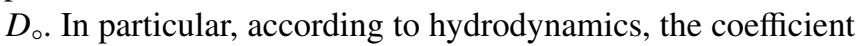
of diffusion perpendicular to a flat solution-solid interface can be represented as ${ }^{6,7}$

$$
D=\frac{6 h^{2}+2 h R}{6 h^{2}+9 h R+2 R^{2}} D_{\text {。 }},
$$

where $h$ is the minimal distance between the particle surface and the solid surface. According to this expression, $D$ is not just numerically smaller than $D_{\circ}$ but it vanishes at $h \rightarrow 0$. Below, we show how this feature can influence the association rate.

To keep the analysis mathematically transparent, we focus on the diffusion-limited association of suspended nanoparticles, $A+B \rightarrow A B$, of radii $R$ and $\rho$ with $R \gg \rho$. In this case, the diffusion coefficient of $A$ is much smaller than that of $B$, and the location of $A$ can be considered to be fixed. The $B$ diffusion coefficient, $D$, appreciably deviates from $D_{\circ}=k_{\mathrm{B}} T / 6 \pi \eta \rho$ only when $A$ is located near $B$. In these regions, the surface of $A$ can be considered to be flat, and accordingly to describe the dependence of $D$ on the distance, $r$, between the centers of $A$ and $B$, one can just replace in Eq. (2) $R$ by $\rho$ and $h$ by $r-\mathcal{R}$, where $\mathcal{R}=R+\rho$. Following this line and aiming, as already noticed, at the transparency of the treatment, we simplify Eq. (2) as

$$
D(r)=\left\{\begin{array}{cl}
(r-\mathcal{R}) \lambda^{-1} D_{\circ} & \text { at } \mathcal{R} \leq r \leq \mathcal{R}+\lambda, \\
D_{\circ} & \text { at } r \geq \mathcal{R}+\lambda,
\end{array}\right.
$$

where $\lambda$ is the parameter comparable with $\rho$ (depending on the taste, one can, e.g., use $\lambda=\rho, 2 \rho$, or $4 \rho$ ). In analogy with Eq. (2), Eq. (3) predicts $D \propto r-\mathcal{R}$ at $r-\mathcal{R} \ll \rho$ and $D=D$ 。 at $r \gg \rho$.

To describe the $A$ and $B$ association, we consider that the $B$ distribution around each $A$ particle is spherically symmetric on average (this is acceptable if the $A$ and $B$ concentrations are not high) and use the steady-state approximation (as it was originally done in Ref. 1). In combination with (3), this approximation yields at $r \geq \mathcal{R}+\lambda$ the following expressions for the B diffusion flux (per an A particle) and concentration:

$$
\begin{gathered}
J=4 \pi r^{2} D_{\circ} \frac{d c}{d r}, \\
c(r)=c_{\circ}-\frac{J}{4 \pi D_{\circ} r},
\end{gathered}
$$

where $c_{\circ}$ is the concentration at $r \gg \mathcal{R}$ (this concentration can be identified with the average $B$ concentration).

At $\mathcal{R}+\delta \leq r \leq \mathcal{R}+\lambda$, the diffusion flux is given by

$$
J=4 \pi r^{2}(r-\mathcal{R}) \lambda^{-1} D_{\circ} \frac{d c}{d r} .
$$

In this expression, $r^{2}$ can be replaced by $\mathcal{R}^{2}$, because $r$ is close to $\mathcal{R}$, i.e.,

$$
J=4 \pi \mathcal{R}^{2}(r-\mathcal{R}) \lambda^{-1} D_{\circ} \frac{d c}{d r} .
$$

To integrate this equation, one should take into account that the decrease of $D$ down to zero at $r \rightarrow \mathcal{R}$ is mathematically correct in the framework of the axiomatic phenomenological hydrodynamics but physically is not acceptable because in this limit the diffusion is influenced by the factors which are ignored in the conventional hydrodynamics. Collectively, this is related to the interface-induced rearrangement of molecules on the length scale $\delta \simeq 1 \mathrm{~nm}$. In the physiological solutions containing, e.g., appreciable amount of water, this rearrangement results in the appearance of the DLVO-type forces including the so-called hydration forces (which are still poorly understood ${ }^{8,9}$ ). Simultaneously, the rearrangement is expected to influence the diffusion in such a way that $D$ remains 
finite at $r \rightarrow \mathcal{R}$. Focusing on the situations with $\rho \gg \delta$, we assume that the diffusion resistance of the narrow region with $\mathcal{R} \leq r \leq \mathcal{R}+\delta$ is negligible and integrate Eq. (7) with the boundary condition $c(\mathcal{R}+\delta)=0$, i.e.,

$$
c(r)=\frac{\lambda J}{4 \pi \mathcal{R}^{2} D_{\circ}} \ln \left(\frac{r-\mathcal{R}}{\delta}\right) .
$$

At $r=\mathcal{R}+\lambda$, the concentrations given by Eqs. (5) and (8) should coincide. This condition yields

$$
c_{\circ}-\frac{J}{4 \pi D_{\circ}(\mathcal{R}+\lambda)}=\frac{\lambda J}{4 \pi \mathcal{R}^{2} D_{\circ}} \ln (\lambda / \delta) .
$$

On the left-hand side of this equation, $\mathcal{R}+\lambda$ can be replaced by $\mathcal{R}$, because $\lambda \simeq \rho \ll \mathcal{R}$. With this tiny modification, Eq. (9) results in

$$
J=\frac{4 \pi D_{\circ} \mathcal{R} c_{\circ}}{1+(\lambda / \mathcal{R}) \ln (\lambda / \delta)}
$$

or

$$
k_{\mathrm{a}} \equiv J / c_{\circ}=\frac{4 \pi D_{\circ} \mathcal{R}}{1+(\lambda / \mathcal{R}) \ln (\lambda / \delta)} .
$$

The latter expression indicates that if the conventional hydrodynamics is used for $D$ at $r$ down to $\mathcal{R}$ (this corresponds to $\delta \rightarrow 0$ ), the association becomes negligible because $k_{\mathrm{a}}$ $\rightarrow 0$. For physically reasonable conditions with $\delta \simeq 1 \mathrm{~nm}$, $k_{\mathrm{a}}$ remains, however, finite. In particular, our treatment has implied $\lambda \ll \mathcal{R}$. In this case, $k_{\mathrm{a}}$ given by Eq. (11) can often be close to that given by Eq. (1).

The reduction of association rate constant (11) compared to that defined by Eq. (1) is physically related to hydrodynamic slowdown of the particle mobility in the contact region. Formally, it can be interpreted as reduction of the effective contact radius (in analogy with the earlier treatment of the effect of the jump length on the association occurring via discrete walks $\left.{ }^{10,11}\right)$.

${ }^{1}$ M. von Smoluchowski, Z. Phys. Chem. 92, 129 (1917).

${ }^{2}$ F. C. Collins and G. E. Kimball, J. Colloid Sci. 4, 425 (1949).

${ }^{3}$ O. G. Berg, Chem. Phys. 31, 47 (1978).

${ }^{4}$ D. T. Gillespie, A. Hellander, and L. R. Petzold, J. Chem. Phys. 138, 170901 (2013).

${ }^{5}$ P. Mereghetti, D. Kokh, J. A. McCammon, and R. C. Wade, BMC Biophys. 4, 2 (2011).

${ }^{6}$ M. A. Bevan and D. C. Prieve, J. Chem. Phys. 113, 1228 (2000).

${ }^{7}$ Y. Kazoe and M. Yoda, Appl. Phys. Lett. 99, 124104 (2011).

${ }^{8}$ V. A. Parsegian and T. Zemb, Curr. Opin. Colloid Interface Sci. 16, 618 (2011).

${ }^{9}$ S. Perni, E. C. Preedy, and P. Prokopovich, Adv. Colloid Interface Sci. 206, 265 (2014).

${ }^{10}$ R. M. Ziff, J. Stat. Phys. 65, 1217 (1991).

${ }^{11}$ R. M. Ziff, S. N. Majumdar, and A. Comtet, J. Chem. Phys. 130, 204104 (2009). 